\title{
Les ruines des jeux olympiques de Grenoble 1968
}

Le tremplin de saut à ski de Saint-Nizier-du-Moucherotte et ses fantômes

The Ruins of the 1968 Grenoble Olympics. The Saint-Nizier-du-Moucherotte Ski Jump and its Ghosts

\section{André Suchet}

\section{OpenEdition}

\section{Journals}

Édition électronique

URL : https://journals.openedition.org/tc/8176

DOI : $10.4000 /$ tc. 8176

ISSN : $1952-420 X$

\section{Éditeur}

Éditions de l'EHESS

\section{Édition imprimée}

Date de publication : 31 octobre 2016

Pagination : 434-447

ISBN : 9782713225291

ISSN : 0248-6016

Référence électronique

André Suchet, «Les ruines des jeux olympiques de Grenoble 1968 », Techniques \& Culture [En ligne], 65-66 | 2016, mis en ligne le 31 octobre 2018, consulté le 29 septembre 2022. URL : http:// journals.openedition.org/tc/8176; DOI : https://doi.org/10.4000/tc.8176 







\section{Les ruines des jeux olympiques de Grenoble 1968}

\section{Le tremplin de saut à ski de Saint-Nizier-du-Moucherotte et ses fantômes}

Proportionnellement au nombre d'études sur les grandes compétitions sportives, la question de la reconversion des équipements construits à cette occasion reste un parent pauvre de la recherche. Alors que plusieurs centaines d'ouvrages et d'articles portent sur la coupe du monde de football, les grands matchs de tennis ou de baseball et surtout les Jeux Olympiques d'été ou d'hiver, les publications qui traitent du devenir des aménagements construits pour l'un de ces grands moments du sport sont à peine quelques dizaines. De plus, le nombre d'études disponibles est comme inversement proportionnel au niveau d'échelle engagé : les recherches à propos des retombées urbaines assez larges d'une manifestation sont peu nombreuses (voir principalement Augustin 2008, Holt \& Ruta 2015, Mangan \& Dyreson 2010, Garcia-Ramon $\&$ Albet i Mas 2000, Capel 2005), mais celles qui traitent en détail des enjeux de reconversion des équipements construits pour une telle occasion sont en nombre carrément insignifiant (Roult \& Lefebvre 2010, Billard 2006, Marsac 2012, Suchet 2013 quasiment exhaustivement). De plus, si les derniers ouvrages relatifs à l'organisation de tels événements présentent un court chapitre à ce sujet - notamment dans le cadre d'une préoccupation de développement durable-, on peut s'étonner qu'il s'agisse d'une préoccupation relativement nouvelle. Autre indicateur des lacunes de la recherche à ce sujet: les dictionnaires de l'urbanisme et de l'aménagement ne consacrent aucune entrée à la reconversion des équipements sportifs (ou installations sportives), alors même que certaines définitions du terme reconversion citent le sport et le tourisme comme outil de revalorisation d'une friche, le plus souvent une friche industrielle (Merlin \& Choay 2005, Wachter 2009, Evert 2010); comme si le sport n'avait pas lui-même ses friches, ses ruines et ses restes! Faisant suite à différentes études à propos des friches touristiques (Bachimon 2013, Gauchon 1996, 1997), ferroviaires (Desmichel 2008, Merzaghi \& Wyss 2009) et minières ou industrielles (Haschar-Noé 2008, Penel 2005, Deshaies 2006) sur lesquelles nous nous sommes appuyé, ce texte traite des friches sportives. 
Les tremplins de saut à ski des Jeux Olympiques d'hiver sont, dans le domaine des friches sportives, un cas d'étude particulièrement heuristique. De Cortina d'Ampezzo 1956 à Turin $2008^{1}$, la presse dénonce chaque fois ces installations si coûteuses qui tombent aujourd'hui en ruine. Il suffit de formuler la recherche «tremplin saut à ski» sur internet pour trouver des dizaines de photographies désolantes de tous ces aménagements construits à travers le monde pour briller, désormais en état de délabrement. Partant d'une géographie sociale et culturelle préoccupée des acteurs, des images et des rapports à l'espace (Di Méo 1998, Gumuchian 1991, Gumuchian et al. 2003) et d'une approche renouvelée du terrain², proche d'une ethno-géographie (Collignon \& Retaillé 2010, Volvey et al. 2012), croisée aux apports d'une approche historiographique du site ${ }^{3}$ pour connaître les faits qui sont à l'origine de ces images et de ces projections d'acteurs, ce travail engage une réflexion sur les ruines des installations sportives.

Cette étude prend appui sur le cas du site de Grenoble, c'est-à-dire le tremplin de SaintNizier-du-Moucherotte ${ }^{4}$, fierté des Jeux Olympiques d'hiver 1968 (Arnaud \& Terret 1993) - une construction de l'architecte et alpiniste français Pierre Dalloz, assisté de l'architecte et sauteur à ski allemand Heini Klopfer (tremplin de $112 \mathrm{~m}$, dit aussi tremplin du Dauphiné ou grand tremplin olympique de Grenoble ${ }^{5}$ ). Le coût total des travaux s'élève vraisemblablement à l'époque à 5,9 millions de francs. Son financement est assuré par l'État à $80 \%$ et par la ville de Grenoble à $20 \%{ }^{6}$. Après les Jeux, l'installation reste en activité une dizaine d'années. Il ne s'agit donc pas du cas le plus éphémère qui soit en comparaison à d'autres installations olympiques qui n'ont servi qu'un ou deux hivers. Plus précisément, comme envisagé au moment des Jeux, le tremplin attire des épreuves nationales, puis des épreuves internationales de la Coupe du monde de saut à ski jusqu'en 1981, mais après cette date aucune activité n'est maintenue sur le site. Faute d'usage la ville de Grenoble qui prévoyait de consacrer 100000 francs chaque année à son entretien décide de ne pas honorer cette prévision. Le syndicat intercommunal, majoritairement contrôlé par Grenoble, créé en 1966 pour s'occuper du tremplin (d'abord du chantier puis de l'organisation des activités) est dissous. La petite commune de Saint-Nizierdu-Moucherotte reste seule propriétaire de l'installation. Soumis à la nature et au temps, le site tombe en ruine et de facto plus aucune activité n'y est possible.

\section{Une impossible reconversion fonctionnelle}

Construire un tremplin homologué de saut à ski dans les Alpes du Nord à une altitude, une exposition et une situation optimale pour permettre la pratique de cette discipline en France n'était pas l'objectif du $\mathrm{COJO}^{7}$ de Grenoble 1968 à Saint-Nizier-du-Moucherotte. L'objectif était d'utiliser cette discipline hivernale pour offrir aux photographes du monde entier une image de Grenoble, capitale des Alpes, métropole de l'innovation au tournant du futur sillon alpin et des anciennes industries du Rhône (Frémont 1987, Debardieux \& Hussy 1996). Le tremplin donnait effectivement l'impression de sauter au-dessus de tout Grenoble. Situé de 1270 
à $1140 \mathrm{~m}$ d'altitude, orienté Ouest, et malgré un effet d'ombre du Moucherotte et des Trois Pucelles (respectivement 1901 et $1456 \mathrm{~m}$ ), le site ne comporte pas un niveau d'enneigement très important. D'ailleurs en 1968, le jour même de certaines épreuves olympiques, la piste du tremplin et sa pente de réception ne sont pas enneigées. Des portages de neige par hélicoptère sont organisés en urgence depuis la Croix de Chamrousse en face (2 $253 \mathrm{~m}$, la station de sports d'hiver de Chamrousse accueillant les épreuves de descente, les trois médailles d'or de JeanClaude Killy). De fait, il ne faut pas chercher pourquoi cette installation n'est pas en usage. Aucun modèle économique et sportif n'est adapté à «remettre en usage ce tremplin » ${ }^{8}$. Cette construction n'a pas été pensée comme une installation sportive régionale de saut à ski mais bien comme un dispositif de médiatisation de la ville de Grenoble et des Alpes du Nord dans le monde (métropole d'à peine 320000 habitants en 1968, de plus de 440000 aujourd'hui), un dispositif quasiment à usage unique. D’ailleurs sa construction même n’a pas été réellement faite pour durer. L'ouvrage n'est planifié qu'au dernier moment et les travaux sont compressés sur six mois, entre juillet 1966 et janvier $1967^{9}$. En réalité, le béton, bien que chauffé au moyen de dispositifs électriques transportables pour aider sa prise, fut travaillé dans des conditions de froid, de pluies et de gel/dégel très néfastes, sa solidité initiale en était déjà réduite.

\section{Une valeur mémorielle sans patrimonialisation}

Cet édifice est une friche sportive, une friche avec ses acteurs publics et privés qui ne s'accordent pas sur son devenir (la municipalité de Saint-Nizier-du-Moucherotte, celle de Grenoble, les riverains du site, le COLJOG ${ }^{10}$, la FFS ${ }^{11}$, l'association Tremplin Saint-Nizier pour la reconstruction d'un nouveau tremplin) et, surtout, ses récits et ses illustres fantômes qui en font un lieu de la mémoire collective grenobloise. Les habitants racontent et se racontent encore la visite de Maurice Herzog, le discours du général de Gaulle ou encore Brigitte Bardot restée bloquée par la neige dans son hôtel du Moucherotte ${ }^{12}$. Des promeneurs pénètrent sur le site, montent les escaliers et s'aventurent dans la structure pourtant très dégradée: des hommes entre 30 et 40 ans par curiosité au détour d'une randonnée et surtout des hommes entre 50 et 70 ans par visite de mémoire. Ils se définissent comme «grenoblois» (mais n'habitent pas de ce côté de la ville) ou «anciens grenoblois» (revenus en séjour sur les lieux de leur «jeunesse», de leurs « années d'études» ou de leur « premier poste comme ingénieur »). Plusieurs d'entre eux, que j’ai rencontrés, suivis et enregistrés, me racontent leur présence aux Jeux Olympiques 1968 ou à une des manches de la Coupe du monde 1980. Plus nombreux encore sont ceux qui «n'étaient pas encore à Grenoble à cette époque » mais qui sont venus voir Alain Prieur, motard de l'extrême et cascadeur des années 1980 sauter du tremplin en octobre 1988 pour un record du monde, un saut à moto de 84 m, sur la structure déjà abandonnée. Ce jour-là, presque comme en 1968, une foule immense est amassée ${ }^{13}$. «Il est mort peu de temps après, en passant d'un avion à un autre sans parachute», me raconte une des personnes. Des récits transformés, arrangés mais 
surtout amplifiés. Le général de Gaulle, Maurice Herzog, Brigitte Bardot, Alain Prieur... tous ces fantômes participent au lieu. Au premier niveau de la structure, sous la tour des juges, des tags et des restes de foyers indiquent aussi la présence d'une jeunesse urbaine des quartiers proches en contrebas ou des rares familles modestes de ce village partagé entre saisonnalité des sports d'hiver et habitat périurbain (Corneloup et al. 2014). À l'inverse des visiteurs précédents, il est difficile de rencontrer et de s'entretenir avec ces jeunes qui viennent certains soirs d'été. D’après deux d'entre eux, pris en dehors du groupe, ils sont plutôt «entre garçons, [...] les filles n'aiment pas ici ». «Des bières», du tabac et vraisemblablement une cigarette roulée de cannabis «qui tourne» constituent l'essentiel du programme de ces soirées. Il s'agit surtout pour eux de se réunir dans un endroit «inhabité», un endroit «comme un repère » (à la fois caché et surplombant), puis aussi un lieu «qui fait peur, surtout la nuit, quand on monte dedans». Si les motivations de ces différents visiteurs peuvent en partie correspondre à une pratique d'urbex (urban exploration), nous n'avons pas rencontré de personnes mentionnant ce terme ou directement impliquées dans ces réseaux communautaires. La municipalité estime à un millier le nombre de visiteurs par an. Une visite interdite qui n'est pas sans risque.

Dès lors, dans quelle mesure ces restes des Jeux d'hiver 1968 ont-ils vraiment quitté le cycle des biens matériels ou immatériels de la société? Dans quelle mesure ces restes olympiques et sportifs ne relèvent-ils pas d'une économie symbolique que pourrait matérialiser un tourisme des friches? Pourquoi aucun processus de patrimonialisation n'est-il en cours, à une époque où tout est fait patrimoine? Sans doute car il s'agit davantage d'une valeur mémorielle que touristique, esthétique, sportive ou même historique. En effet, ces cathédrales abandonnées exercent un sentiment antinomique de répulsion et d'attirance de nature à entretenir une mémoire collective d'un événement temporaire, une mémoire vive. Or on savait déjà que mémoire et patrimoine ne faisaient pas forcément bon ménage dans le cas d'un passé douloureux ${ }^{14}$, ce travail concourt à montrer qu'il en est de même d'un passé bienheureux comme une réussite nationale olympique et sportive datée des Trente Glorieuses.

\section{Conclusion et perspectives}

Restes fantômes d'une gloire sportive, économique et géopolitique plus ou moins teintée de nationalisme, ce tremplin olympique des Jeux de Grenoble 1968 construit à Saint-Nizier-duMoucherotte, comme beaucoup d'autres sites de saut à ski dans le monde, n'a eu que peu d'usage en dehors de sa raison d'être olympique. Ces édifices ne sont quasiment jamais détruits. On leur attribue instinctivement une valeur importante sans pour autant qu'ils fassent l'objet d'une politique de patrimonialisation dans la mesure où il s'agit davantage d'une valeur mémorielle vive que d'un objet passé désormais éteint qui n'attend que sa mise en tourisme. Impossibles à requalifier ou à reconvertir malgré les promesses et les projets, ces installations olympiques mettent en tension la notion de temporalité, interrogent la dimension pratique et symbolique 
de l'aménagement de l'espace et soulèvent au final les enjeux politiques, sociaux, économiques, environnementaux et paysagers du rapport entre mémoire et patrimoine.

Dans ce débat qui n'est pas seulement théorique, à contre-courant des expertises professionnelles qui tentent de multiplier les initiatives de reconversion du site plus ou moins pertinentes (transformation de la tour des juges en restaurant d'altitude, du tremplin en mur d'escalade, organisation d'une base de VTT de descente, de la tribune en salle d'exposition) ${ }^{15}$, cette étude interroge in fine la pertinence du scénario ruskinien de gestion publique et privée d'un édifice, c'est-à-dire un abandon accompagné, sécurisé mais aussi valorisé.

«Dans Les Sept lampes de l'architecture (1900), John Ruskin, historien de l'art britannique, compare le patrimoine avec un être humain qu'il faut soutenir (en le restaurant le moins possible) mais qu'il faut aussi laisser mourir. Même si l'on doit veiller avec vigilance sur un vieil édifice, il faut accepter que sa dernière heure sonne "mais qu'elle sonne ouvertement et franchement, et qu'aucune substitution déshonorante et mensongère ne le vienne priver des devoirs funèbres du souvenir". Ruskin prône avec une ferveur militante, la "non-restauration". Si cette approche peut paraître surprenante ou trop "romantique", cette option d'aménagement est très nettement développée dans l'Europe du Nord, contrairement à la France encore très imprégnée des théories de Viollet-le-Duc.

[On peut seulement citer] le cas du lavoir à charbon de Chavannes [avec] un choix à contre-courant de la doctrine traditionnelle des monuments historiques. L'immense bâtiment de $8000 \mathrm{~m}^{2}$, haut d'une douzaine d'étages, est resté presque intact, de même que son intérieur encore rempli des machines et des outils servant au tri et au lavage du charbon. Les locomotives abritées dans les hangars, les wagons garés sur les faisceaux de voies ferrées au cœur d'une emprise de 32 hectares gagnée par les acacias. La communauté urbaine de Montceau-les-Mines n'a pas opté pour un quelconque projet de réhabilitation en pôle de logements et bureaux (pas le besoin), ni pour un grand équipement de loisir (trop coûteux à gérer) mais a retenu le projet du cabinet d'architectes hollandais MVRDV consistant à laisser la nature reprendre possession du site en friche tout en veillant attentivement à en maîtriser l'évolution. L'objectif proposé par ce cabinet en 2004 est d'inscrire le lavoir abandonné dans le territoire, d'en faire un repère symbolique, de ritualiser l'inévitable dépérissement de cet objet industriel. [...] Ce choix de la sanctuarisation, qui a séduit les acteurs de cette communauté urbaine, est aussi un choix économique (ce projet était dix fois moins coûteux que les autres). »

Le scénario ruskinien de gestion d'un équipement inusité, extrait de Desmichel, 2008.

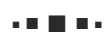



afin de donner l'impression de sauter au-dessus de la ville de Grenoble.

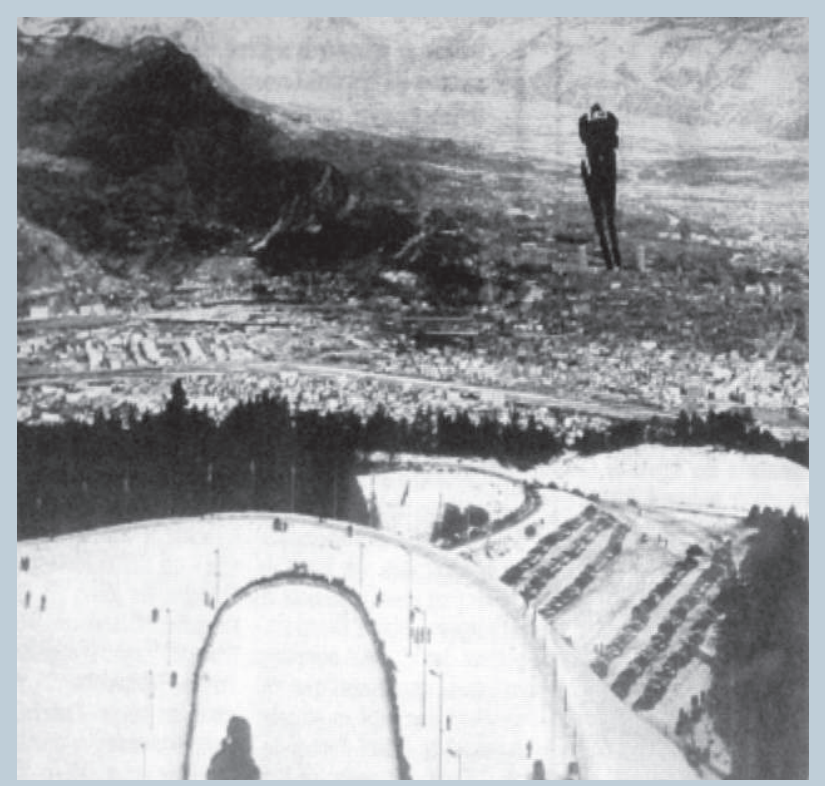

2. La foule pendant les entraînements officiels en 1968, puis la visite de Maurice Herzog. Plus encore, les manches de finale attirent près de 70 ooo spectateurs et reporters.

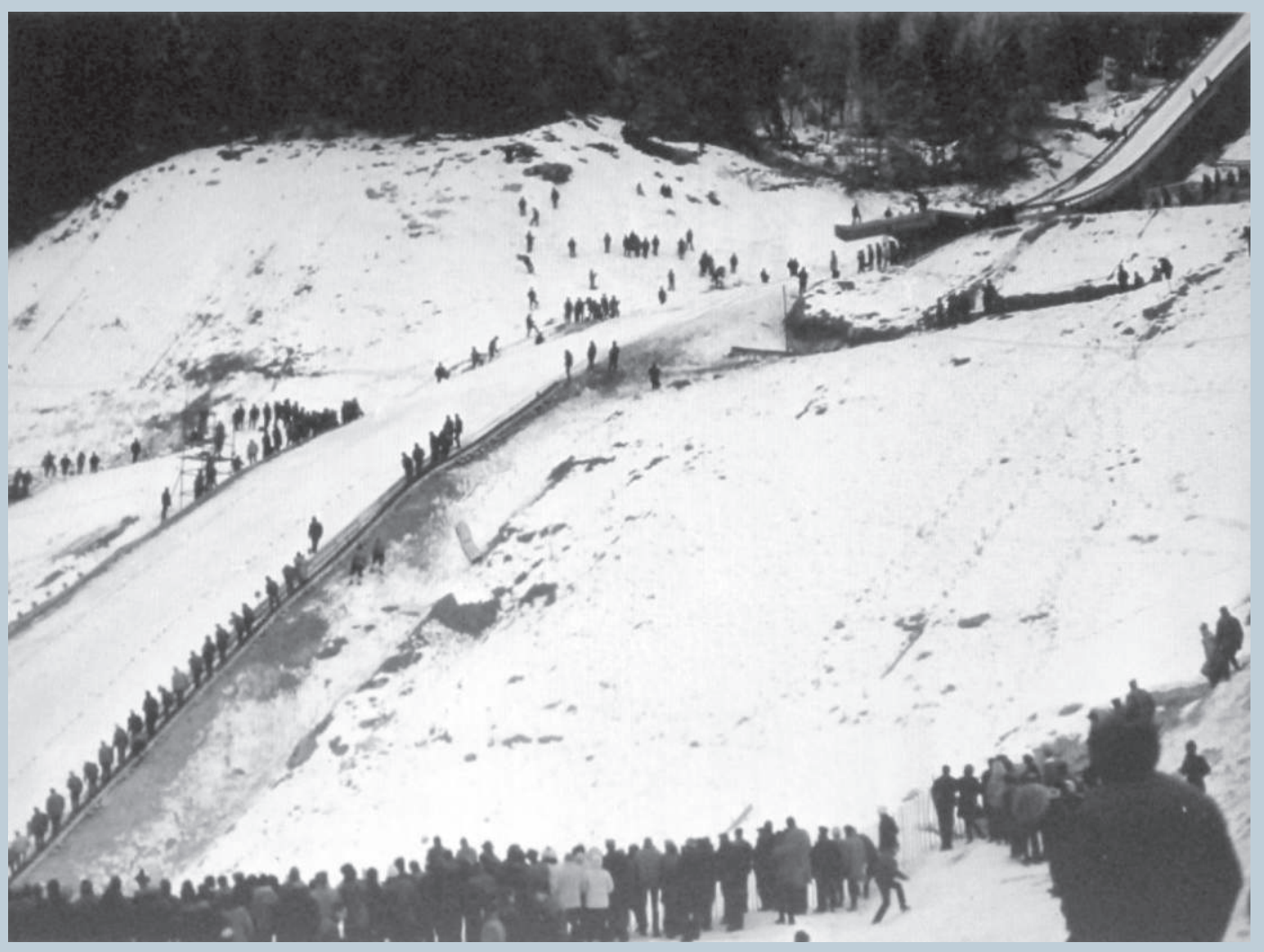




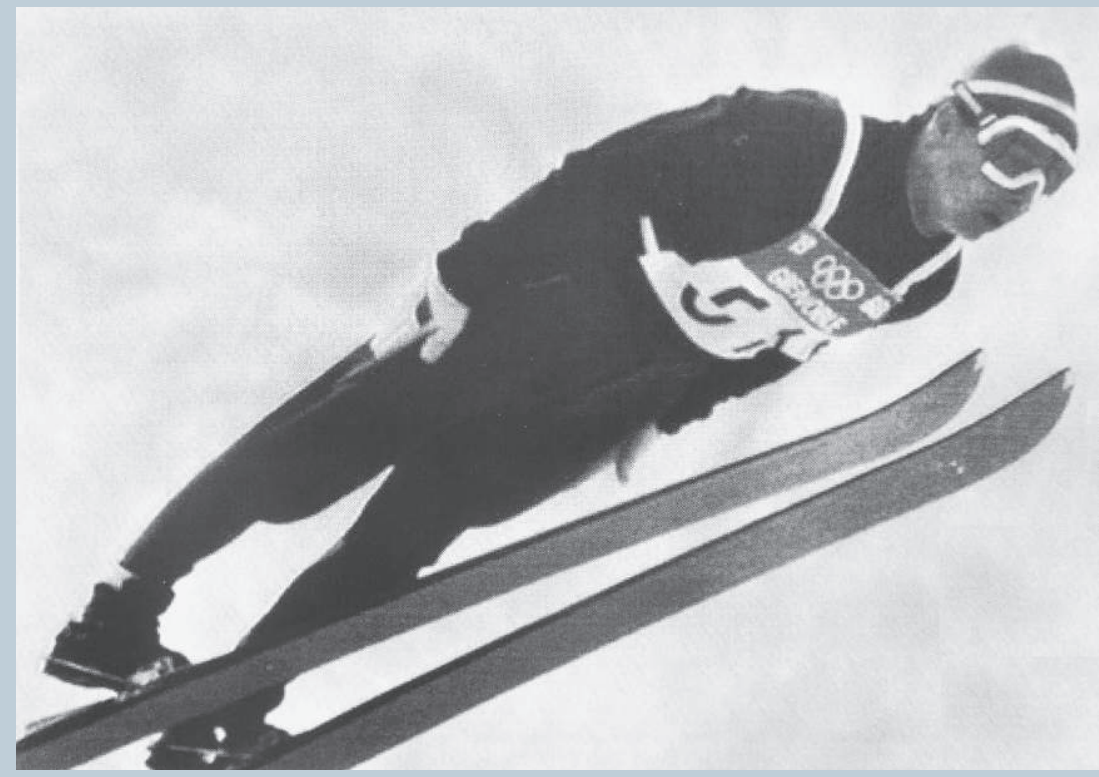

3. Jiri Raska, sauteur tchécoslovaque double médaillé olympique en 1968 sur le tremplin de Saint-Nizier-du-Moucherotte.

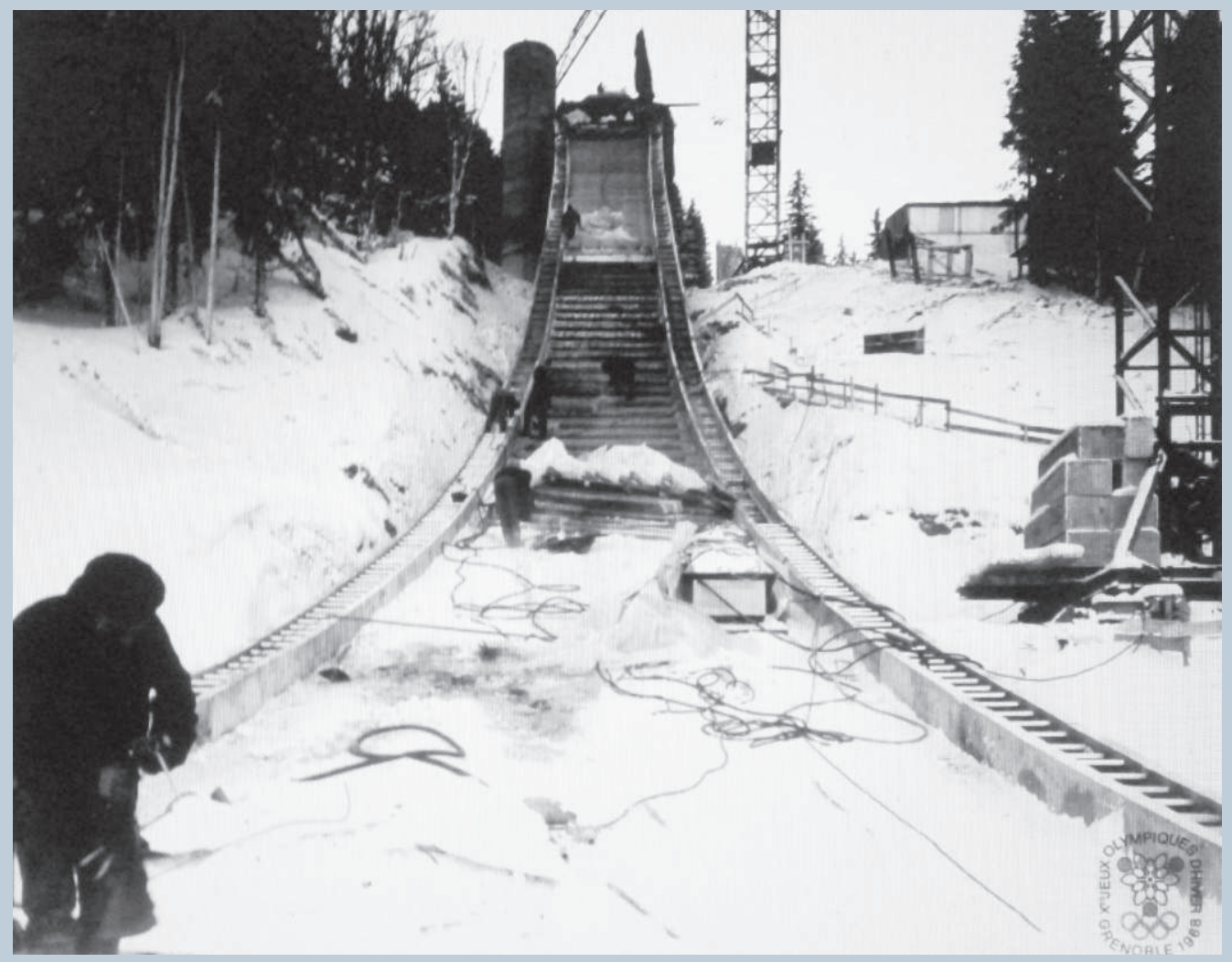

4. Le chantier de construction du tremplin au cours de l'hiver 1966, des conditions qui diminuent les capacités du béton. 


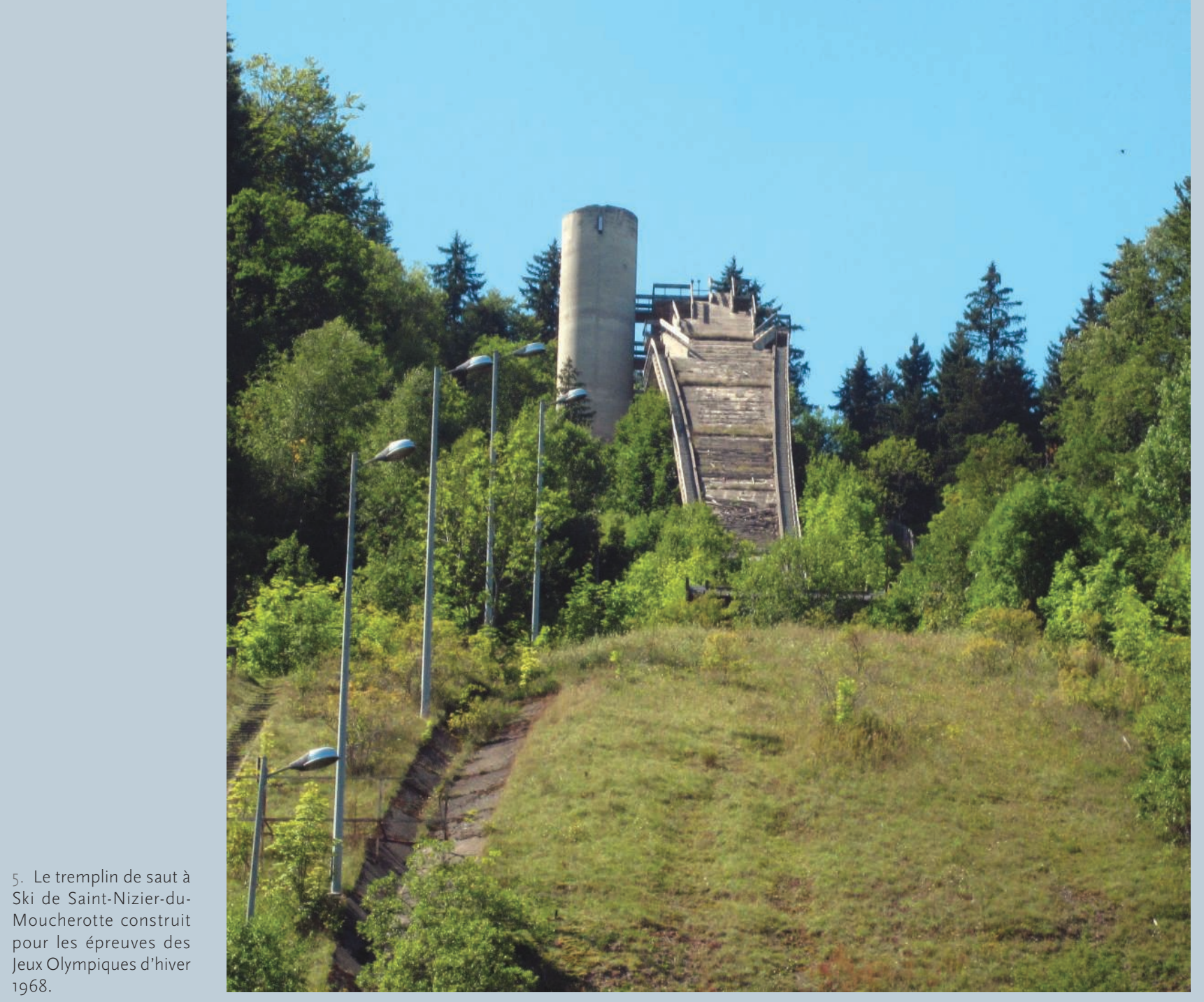




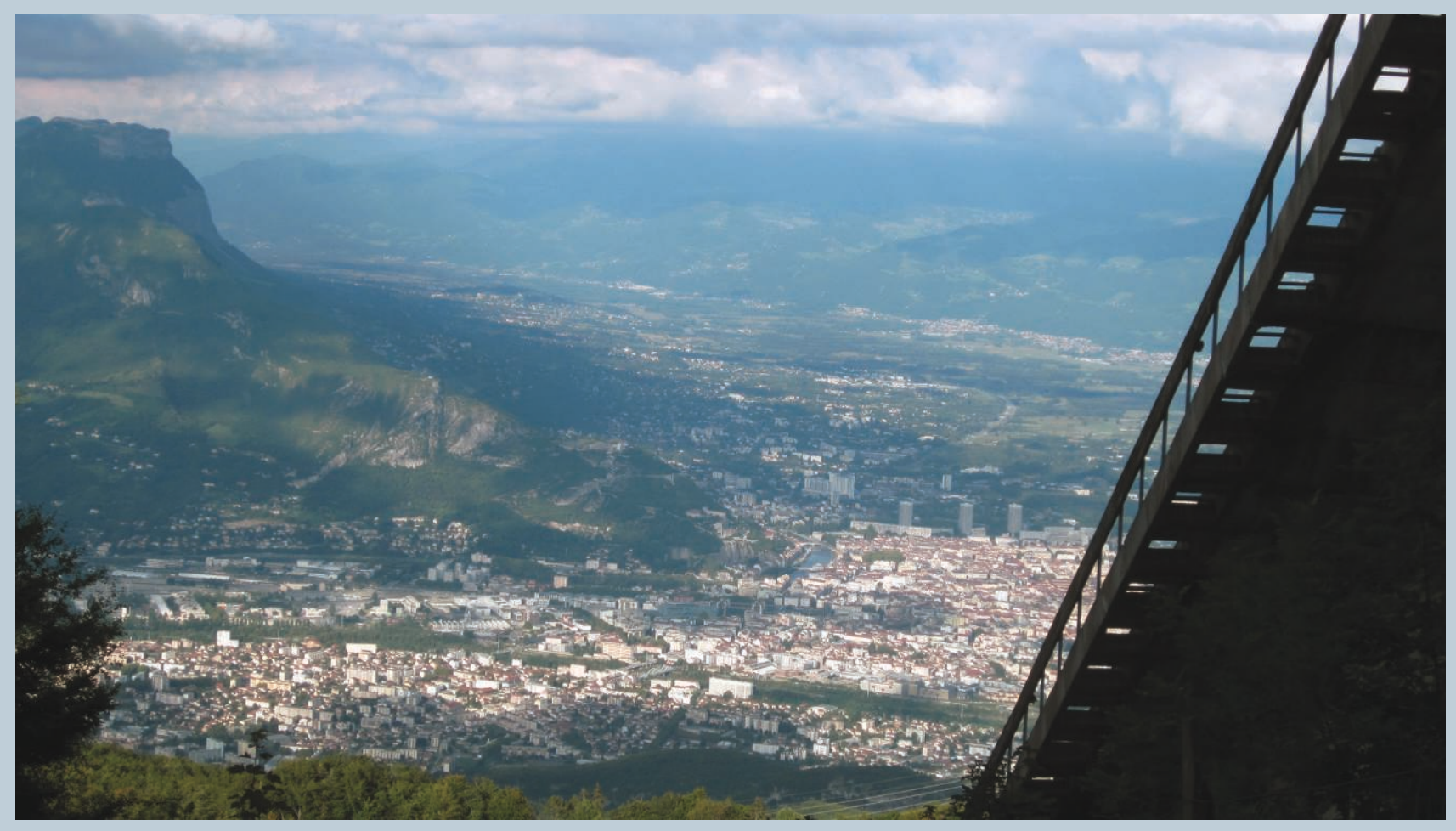

6. Le tremplin au-dessus de toute l'agglomération grenobloise. On peut reconnaître, peu avant la confluence de l'Isère et du Drac, le début de la Presqu'île scientifique qui abrite plusieurs établissements de haute technologie, dont un centre du CEA. Partiellement dans l'ombre du massif de la Chartreuse, on peut identifier le centre-ville relié à la Bastille du mont Jalla par un téléphérique et délimité en quelque sorte de l'île-Verte par les deux barres et les trois tours de $98 \mathrm{~m}$ construites entre 1963 et 1967. Plus loin, on remarque l'hôpital Michallon inauguré en 1975 et, à peine dissimulé ici par la rampe du tremplin, le Stade de glace des Jeux 1968 devenu le Palais des sports de la ville. Enfin se trouve le campus universitaire développé à partir de 1961, puis en remontant l'Isère vers Chambéry, dans cette vallée du Grésivaudan autrefois connue pour son agriculture, ses industries du papier et son hydroélectricité (la houille blanche), se multiplient désormais, sous le nom d'Inovallée, les industries de pointe, SSII et autres startups que dominent les résidences de ces nouveaux dirigeants d'entreprise. 


\section{Notes}

1. Francesco Pastorelli «Les ruines de Turin », Alpenscène CIPRA, 2010 : 94: 16-17. Alberto Custodero, «Turin et son site Olympique fantôme», Courrier International 1004, 28 janvier 2010 (reproduit de La Repubblica, 5 janvier 2010).

2. Étude de terrain réalisée de manière discontinue entre 2010 et 2013 en marge d'une thèse de doctorat en géographie à propos des dynamiques sportives de nature et du tourisme de montagne dans les Pyrénées au regard de la situation dans les Alpes, thèse préparée à l'Institut de géographie alpine de l'université de Grenoble, anciennement université Joseph Fourier (Suchet 2012).

3. Archives municipales de Grenoble (principalement sous-série 3R et série M); archives municipales de Saint-Nizier-du-Moucherotte (boites 1w29, 1w30 et lw31); documentation du COLJOG; le reportage télévisé L'avenir du tremplin olympique à Saint Nizier du Moucherotte, archives INA, FR3 (collection: JT Rhône-Alpes soir) 8 nov. 1998, et site internet www. jo-grenoble.fr/grenoble-olympique/.

4. Le tremplin se trouve sur la commune de Saint-Nizier-du-Moucherotte (1 083 habitants, 998 permanents) qui lui donne son nom d'usage. Sur les dernières décennies, cette bourgade du massif du Vercors, toute proche de Grenoble, entourée des stations de sports d'hiver très actives de Villard-deLans et Lans-en-Vercors, est passée géographiquement d'une fonction touristique (autrefois station climatique et station de sports d'hiver dont il ne reste que peu d'équipements) à une fonction résidentielle d'habitat périurbain de Grenoble agglomération (voir à ce sujet Corneloup et al. 2014).

5. Le petit tremplin ( $90 \mathrm{~m}$ ) des épreuves olympiques est construit simultanément à Autrans (ainsi que deux tremplinsécole de 15 et $25 \mathrm{~m}$ plus un tremplin intermédiaire de $60 \mathrm{~m}$ ). Le site, prévu pour un usage sportif régional, continue d'être utilisé été comme hiver, notamment par le pôle espoir de la FFS. Voir à ce sujet: Communiqué du maire Gabriel Tatin, Les tremplins au coeur des préoccupations, www.autrans.fr/tremplins.html.

6. Le remboursement de cette somme ne s'achève que 30 ans plus tard, en 1995.

7. Comité d'Organisation des Jeux Olympiques (COJO).

8. En 1990, une étude sur le devenir du site aboutit à la conclusion que les bétons doivent être remis en état dans les 5 ans, après quoi l'état de corrosion des structures sera irréversible et la réhabilitation du tremplin ne sera plus possible (réhabilitation estimée à 15,96 millions de francs). Différentes propositions sont alors faites, mais laissées sans suite. La démolition chiffrée à 2,88 millions de francs est aussi écartée. Certains accusent l'attitude du maire de Grenoble Alain Carignon, d'autres celle d'un riverain qui s'est opposé, à différentes reprises, aux activités du site. Au final rien n'est fait.

9. COJO 1968, Xe Jeux Olympiques d'hiver (Grenoble 1968) : rapport officiel, 1969, p. 95.

10. Conservatoire Observatoire Laboratoire de Jeux Olympiques de Grenoble (COLJOG).

11. Fédération française de ski (FFS).

12. Un hôtel, quasiment mythique, fut construit en 1959 au sommet du Moucherotte, par un notaired'Aix-enProvence, Jean Zucchetta. L'établissement situé sur un promontoire rocheux, totalement isolé, n'était accessible que par un téléphérique. Dans l'ambiance des années 1960, cet hôtel de luxe attire les plus grandes stars comme Dalida, Luis Mariano, Claude Brasseur, Mireille Darc et Brigitte Bardot. Il servira de cadre principal au film de Roger Vadim, La Bride sur le cou, 1961. Faute de rentabilité, il ferme en 1975, le téléphérique est démantelé quelques années plus tard et une démolition totale du bâtiment est effectuée en 2001.

13. Voir à ce sujet: J.-L. Otero de Saavedra Mira, Alain Prieur (1949-1991) Francia, pilotos-muertos. com/2012/Prieur\%20Alain.html.

14. La mise en tourisme des camps de concentration et d'extermination de l'Allemagne nazie, la valorisation des vestiges d'un drame, la visite des cimetières, ne sont pas sans poser débat (voir à ce sujet Buntman 2008, Lennon \& Foley 2000).

15. L. Grima, 1998 Vers la reconversion du grand tremplin des Jeux Olympiques de Grenoble - St-Nizier-du-Moucherotte. Mémoire de DESS, université Joseph Fourier. J. Vuillermet, Le tremplin Olympique de saut à ski: enjeu de développement touristique durable pour Saint-Nizier-du-Moucherotte, mémoire de DESS, université Joseph Fourier 2003. Projets et démarches de l'association Tremplin Saint-Nizier pour la reconstruction d'un nouveau tremplin à cet endroit, et site internet: jo-grenoble.fr/ tremplin-olympique-1968/\#more-1530. 


\section{I'auteur}

André Suchet est docteur en géographie de l'Institut de géographie alpine, à l'UMR PACTE de l'université de Grenoble, et actuellement post-doctorant CMIRA au Département de Géographie de l'université Rovira i Virgili, dans l'équipe GRATET, à Tarragone en Espagne. Ses recherches portent sur les dynamiques territoriales autour du sport, du tourisme et des loisirs.

\section{Iconographie}

Image d'ouverture. L'afficheur de résultat. (C) A. Suchet. 1 et 2. (C) COJO, Grenoble, 1968.

3. Anonyme, Grenoble, 1968.

4. (C) Mairie de Saint-Nizier-du-Moucherotte, 1967.

5 et 6 . () A. Suchet.

\section{Références}

Arnaud, P. \& T. Terret 1993 Le Rêve blanc. Olympisme et sports d'hiver en France. Bordeaux: Presses universitaires de Bordeaux.

Augustin, J.-P. 2008 « Installations olympiques, régénération urbaine et tourisme», Téoros 27 (2) : 31-35.

Bachimon, P. 2013 Vacance des lieux. Paris: Belin.

Billard, G. 2006 «Après la compétition olympique, le village urbain durable. Lapport des jeux d'été de 2000 à Sydney, Australie», Les Annales de la recherche urbaine 101 : 100-107.

Buntman, B. 2008 « Tourism and Tragedy: The Memorial at Belzec, Poland», International Journal of Heritage Studies 14 (5): 422-448.

Capel, H. 2005 El modelo Barcelona: un examen crítico. Barcelona: Serbal.

Collignon, B. \& D. Retaillé dir. 2010 Le terrain. Numéro thématique de L'Information géographique 74 (1).

Corneloup,J., Bourdeau, P., Bachimon, P. \& O. Bessy 2014 «L'habitabilité récréative périurbaine (Autour du cas de Saint-Nizier-du-Moucherotte, agglomération de Grenoble)», Sociétés 125: 47-58.

Debarbieux, B. \& C. Hussy 1996 «Le nouveau "sillon alpin" : territoire en gestation ou invention technocratique?», Mappemonde 11 (1): 40-45.

Deshaies, M. (dir.) 2006 Réhabilitation et reconversion des espaces industriels et urbains dégradés. Numéro thématique de la Revue géographique de l'Est 46 (3-4).

Desmichel, P. 2008 «La gare monumentale de Canfranc à l'épreuve des temps. Grandeur et décadence d'un patrimoine ferroviaire de la montagne aragonaise (Espagne)», Cybergeo: revue européenne de géographie, cybergeo.revues.org/18342.

Di Méo, G. 1998 Géographie sociale et territoires. Paris: Nathan.

Evert, K.-J. dir. 2010 Encyclopedic dictionary of landscape and urban planning. Berlin: Springer. 2 vol.

Frémont, A. 1987 «Milieu géographique et innovation: le cas grenoblois », Revue de géographie alpine, 75 (4): 293-310.

Garcia-Ramon, M.-D. \&A. Albet i Mas 2000 «Pre-Olympic and post-Olympic Barcelona, a "model" for urban regeneration today? » Environment and Planning $A$ 32(8): 1331-1334.

Gauchon, C. 1997 «Anciennes remontées mécaniques dans les montagnes françaises: pour une géographie des friches touristiques », Bulletin de l'association de géographes français 74 (3): 296-310.

— 1996 «Friches touristiques: les anciennes grottes touristiques en France » in Actes du Symposium international "Grottes aménagées et contrôle de l'environnement souterrain”. Frabosa Soprana: 11-20.

Gumuchian, H. 1991 Représentations et aménagement du territoire. Paris: Anthropos.

Gumuchian, H., Grasset, E., Lajarge, R. \& E. Roux 2003 Les acteurs, ces oubliés du territoire. Paris: Anthropos.

Haschar-Noé, N. 2008 «Action publique et reconversion durable d'une friche industrielle. L'exemple de Cap'Découverte» in O. Bessy dir. Sport, loisir, tourisme et développement durable des territoires. Voiron: Presses universitaires du Sport: 109-122.

Holt, R. \& D. Ruta dir. 2015 Routledge Handbook of Sport and Legacy Meeting the Challenge of Major Sports Events. Londres: Routledge.

Lennon, J. \& M. Foley 2000 Dark Tourism: The Attraction of Death and Disaster. Londres: Continuum. 
Mangan, J.A. \& M. Dyreson dir. 2010 Olympic Legacies: Intended and Unintended. Londres: Routledge.

Marsac, A. 2012 «Accueil des épreuves olympiques de slalom en canoë-kayak et développement territorial (1972-2012)», Revue européenne de management du sport 36: 59-72. www.acteursdusport.fr/199-rems.htm

Merlin, P. \& F. Choay dir. 2005 Dictionnaire de l'urbanisme et de l'aménagement. Paris: Presses universitaires de France.

Merzaghi, F. \& M. Wyss 2009 «Comment une friche ferroviaire se transforme en quartier durable: Le quartier Écoparc à Neuchâtel en Suisse ». VertigO 9 (2), vertigo. revues.org/8757.

Penel, G. 2005 «Le sport comme moyen de réactiver un territoire en crise. L'exemple de la station de ski de Nœux-les-Mines». Revue européenne de management du sport 13 : 57-76.

Roult, R. \& S. Lefebvre 2010 «Planning and Reconversion of Olympic Heritages: The Montreal Olympic Stadium », International Journal of the History of Sport 27 (16-18) : 2731-2747.
Ruskin, J. 1900 La Couronne d'olivier sauvage. Les Sept lampes de l'architecture. Paris: Société d'Éditions Artistiques.

Suchet, A. 2013 «La reconversion des équipements d'une grande compétition sportive de nature. Désorganisation, stratégies d'acteurs et conflits d'intérêts autour de l'héritage matériel des Jeux pyrénéens de l'aventure 1993», Revue européenne de management du sport 40 : 65-76, www.acteursdusport.fr/199-rems. htm.

- 2012 Acteurs, gouvernance et dynamiques de projet dans la concurrence des territoires en tourisme. Étude autour du programme sportif «Pyrénées: Laboratoire de l'Olympisme» 1988-1994 et des Jeux Pyrénéens de l'Aventure 1993.Thèse de Doctorat, université de Grenoble.

Volvey, A., Calbérac, Y. \& M. Houssay-Holzschuch dir. 2012 Terrains de je. (Du) sujet (au) géographique. Numéro thématique des Annales de Géographie 121 (687-688).

Wachter, S. dir. 2009. Dictionnaire de l'aménagement du territoire. Paris: Belin.

\section{Pour citer cet article}

Suchet, A. 2016 «Les ruines des jeux olympiques de Grenoble 1968. Le tremplin de saut à ski de Saint-Nizier-duMoucherotte et ses fantômes», TechniquesECulture 65-66 «Réparer le monde. Excès, reste et innovation », p. 434-447. 
